\title{
Connective Ethnography for the Exploration of E-Science
}

Author's accepted draft. Published as Hine, C. 2007. Connective ethnography for the exploration of e-science. Journal of Computer Mediated Communication.12(2) http://jcmc.indiana.edu/vol12/issue2/hine.html

\begin{abstract}
E-science comprises diverse sites, connected in complex and heterogeneous ways. While ethnography is well established as a way of exploring the detail of the knowledge production process some strategic adaptations are prompted by this spatial complexity of e-science. This paper describes a study which focused on the biological discipline of systematics, exploring the ways in which use of a variety of information and communication technologies has become a routine part of disciplinary practice. The ethnography combined observation and interviews within systematics institutions with mailing list participation, exploration of web landscapes, and analysis of expectations around information and communications technologies as portrayed in policy documents. Exploring connections between these different activities offers a means to understanding multiple dimensions of e-science as a focus of practice and policy. It is important when studying e-science to engage critically with claims about the transformative capacity of new technologies and to adopt methodologies which remain agnostic in the face of such claims: a connective approach to ethnography offers considerable promise in this regard.
\end{abstract}

\section{Introduction}


This paper describes a methodological response to e-science which builds on ethnographic traditions for understanding scientific practice. As applied to science, ethnography has offered a way of delving beneath surface-level accounts of the knowledge making process. A swathe of influential laboratory-based ethnographies initially helped to establish a view of science as a constitutively social practice (H. M. Collins, 1975, 1985; Knorr-Cetina, 1981; Latour \& Woolgar, 1986; Lynch, 1985; Zenzen \& Restivo, 1982). Upon this basis contemporary studies range more widely (Heath, 1998; Heath, Koch, Ley, \& Montoya, 1999; Hess, 2001; Martin, 1994, 1998), inspired in part by the renewed focus within anthropology on ethnography as a multi-sited endeavour and a means to explore global connections suffusing local sites (Amit, 2000; Burawoy, 2000; Gupta \& Ferguson, 1997; Marcus, 1995, 1998). Recent science studies adopt quite diverse notions of the appropriate field site for developing an understanding of science. Knorr-Cetina has explored the prevalence of different scientific cultures by comparing laboratories in diverse disciplines (Knorr-Cetina, 1999) and large scale historicethnographic studies provide pictures of the development of disciplinary cultures over time (H. Collins, 2004; Galison, 1997). Studies of field science (Henke, 2000) and of science manifested in diverse arenas of public life (Epstein, 1996) broaden attention out from the laboratory, whilst studies such as Mody's (2005) work on the role of sound in scientific practice narrow the focus down to a specific issue within the laboratory. Laboratory ethnographies have offered an iconic exemplar for science studies in their close attention to scientific practice as a site of social action, but they far from monopolize the theoretically interesting ways to define the field. 
Building on this openness to multi-sited science, and to structures of meaning-making which do not conform to laboratory boundaries, science studies would appear to be well placed to pursue ethnographic understandings of e-science. E-science offers the opportunity to build on existing understanding of the significance of communicative structures for the practice of science (Latour \& Woolgar, 1986), appreciation of the multidimensional practices which solidify new communication regimes (Hilgartner, 1995; Hilgartner \& Brandt Rauf, 1994) and awareness of the importance of examining classificatory schemes as they become embedded in new infrastructures (Bowker, 2000). New questions also come to the fore, focused around the extent to which distributed scientific practice reshapes knowledge production processes and outcomes, and the degree to which developments are experienced differently across diverse disciplines (Hine, 2006). New potential field sites also come into focus, as science is practiced not just in laboratories, but in computer science departments, across networks, within distributed databases and via information infrastructures. Identifying appropriate field sites and exploring the connections between them may require some imaginative strategies on the part of ethnographers, who will need to accept that a hyperlink in a database, just as much as a conference presentation or a conversation over the microscope constitutes a form of scientific practice (Beaulieu, 2005; Beaulieu \& Simakova, 2006).

The specific approach to ethnography taken in this paper orients towards connection and mobility rather than to pre-defined field sites. In this formulation virtual ethnography (Hine, 2000) takes an interest in varied forms of computer-mediated communication as providing for cultural activities amenable to ethnographic exploration in their own right. 
This form of ethnography moves between online and offline as users of computermediated communication do (Leander \& McKim, 2003) looking at the construction of boundaries and the ways in which different forms of communication are used to contextualize one another. It is open to embedding processes, looking both at the ways that lives are embedded into computer-mediated communication and processes through which computer-mediated communication is embedded into lives (Howard, 2004). This paper describes a case study which took a connective approach to ethnography of escience, describing three different strategies for pursuing connection which emerged as important in this particular case.

Whilst exploring the mutual elaboration between online and offline activities is important, as Leander and McKim (2003) describe, it proved significant in this case also to track connections between activities and expectations about those activities. E-science has been a site of intense policy interest and funding intervention, and it proves important to explore the particular ways in which these concerns shape e-science as a cultural artefact (Hine, 2000) with specific connotations for those involved. Commentaries on the e-science phenomenon have knock-on effects both on what people find it meaningful to do with information and communication technologies, and on what is in turn done with those activities. Online and offline may well not be categories that participants orient to in the context of their efforts to sustain fundable activities which are respected by their peers and their institutions. Once again, tracing the relevant connections and boundaries is an ethnographic puzzle rather than a question to be solved before beginning the ethnography. 


\section{Connective practices in e-science}

The study described here focuses on the discipline of systematics, the branch of biology concerned with the classification and naming of organisms and the exploration of relationships between them. In recent years systematics has made substantial use of computer-mediated communication and has also given rise to numerous initiatives to make resources available on the internet, both to a professional systematics community and to a wider public of users of information on biological diversity. Contemporary systematics is not, strictly, e-science according to most definitions (Hey \& Trefethen, 2002), most notably in that high-end computation is largely absent. Systematics does however share with e-science a concern with access to communal data resources.

Systematists have had to face many of the issues which trouble e-science implementations, including the design of data structures, development of standards and emergence of intellectual property concerns. These issues have arisen in the context of ongoing concerns about the funding and reputation of the discipline, and a high profile role in the global politics of biodiversity conservation.

The ethnography visited a diverse array of online and offline sites. Over a period of three years, informed by a previous long term engagement with the discipline (since 1986), I visited several institutions, conducted interviews with systematists and participants in database initiatives at these sites and toured museums and specimen collections. I also conducted extensive online fieldwork, exploring web sites and hyperlink landscapes, participating in online forums and conducting email interviews. The aim was a holistic 
understanding of the disciplinary scene which made specific uses of information and communication technologies into meaningful practices for participants to engage in. The research design, in effect, emerged in the process of the ethnography and to a large extent was its product rather than its precursor. Some of the ethnography was more conventionally organized around sites that could be identified in advance: one would not, for example, expect credibly to explore systematics without visiting at least some of the major institutions where the work is done. Other connections came into visibility as the project progressed, when drawn on by interviewees and mailing list members in their accounts or uncovered in literature searches and explorations of the online landscape. Relevance is not always obvious in advance, and so this approach involves a certain amount of tentative exploration of potential research directions.

In this paper it is not the intention to give an account of the entire ethnography. I will focus instead on three aspects which have a particular methodological significance in illustrating connective ethnographic strategies to pursue e-science. The first of the following sections examines one aspect of the connections between online and offline, focusing on the particular issue of how far observing an online forum gives us a means to understand the concerns of the discipline as a whole. The second section pursues online/offline connections again, this time focusing on the variable visibility of institutional structures within online landscapes, and highlighting one tactic for raising questions about connections between online and offline structures. The third section then briefly articulates the importance of pursuing connections between activities and expectations, looking at the presentation of expectations for online activities in the policy 
domain, and the packaging of online activities for consumption within that domain. The conclusion then examines the extent to which these strategies transform the ethnographic project, and discusses their applicability to other domains both within e-science and beyond.

\section{Online forums as disciplinary venues}

One key starting point for understanding and experiencing contemporary systematics was a mailing list. The Taxacom list began as a bulletin board in 1987, and whilst originally a list for discussion of issues related to computing in systematics it became over the years a more general purpose forum hosting announcements and job advertisements, appeals for materials and for advice, and discussions on "hot topics" in the discipline. Throughout my engagement with the field I observed this active and popular list as a way of keeping in touch with what the discipline was up to. New initiatives were often promoted here, and sometimes heated discussions broke out relating to controversial topics. I found myself using the mood of the list as a means to judge the reaction of systematists to proposals that I was reading in the literature. I also used the list quite specifically to track some particular issues that were relevant for my research, drawing on list archives that were available online going back to 1991 . I was interested in the way that computing skills had been progressively integrated into the job descriptions of museum and herbarium curators and I used job advertisements sent to the list over the years as a source of data to track developments in this area. I was also able to use the list archive to 
pinpoint the early acceptance of images as a way of identifying unknown specimens at a distance.

The list was therefore a considerable practical aid to the observation of "the discipline", as a complement to conversations that I was already having with individual interviewees face-to-face about their involvement in online initiatives. It is, however, not safe to treat the list as a transparent mirror of disciplinary concerns. In particular, we do not know whether the members of the list are representative of the discipline as a whole, nor do we know whether contributions on the list do represent a fair portrayal of the kind of concerns that might be discussed in other locations. We have no way of judging whether the majority of readers agree with points being made, even though silence on a mailing list often appears as if it is acquiescence (Hine, 2000). As a point of methodological rigour it therefore proved important to explore the extent to which the list could plausibly be considered reflective of the discipline. One means of assessing this point was to discuss the list with the interviewees that I met face-to-face, asking whether they used the list and what it meant to them.

By asking about the list face-to-face I began to find out about diverse perspectives on its status. Some interviewees were avid users, but most had some reservations about the usefulness of the list for example because it consumed too much time, or it overemphasized particular topics or because it was dominated by a few vocal personalities. Subscribers told me how they passed items on to relevant colleagues, and non-subscribers described being recipients of snippets of list content referred by peers. Content moved 
beyond the boundaries of overt list membership, and readers appeared to actively consume and to question what they read. It was clear that the list could not, therefore be taken straightforwardly to represent the way the discipline thought.

In order to extend my assessment of the list as a reflection of disciplinary concerns I deployed the list itself, posting a message asking for assistance in finding out what the list meant to its users. An initial set of questions invited respondents to tell me about their list subscription, about the other lists they subscribed to and about how well they felt the list reflected the concerns of the discipline. As responses came in I replied to each individually, asking further questions of detail and clarification. Respondents gave me insights into how they read messages and what they thought list biases might be. I particularly encouraged "lurkers" to respond and several did so, explaining how they monitored the list and why they felt it inappropriate to participate by sending messages.

Opinions of the list were, as might be expected, diverse. However, some common threads emerged. In particular, it became clear that while participants used their list membership as a way of monitoring the concerns of the discipline, just as I was doing, there were also a range of selective reading practices that they used to filter what they were reading. These included recognition that various vocal participants had particular issues that they championed, and that these positions were therefore over-represented. Controversial issues were particularly likely to feature in list discussions. The list was also felt to be skewed towards the concerns of the USA. Some felt that the list also demonstrated a gender bias, with few women sending messages despite a considerable number of women 
both working in the discipline and subscribing to the list. Respondents varied in the contrasts that they drew and the strands of continuity that they pointed to between the discipline and the list. However, whilst the list was important to them as a way of monitoring their discipline, for many it was also felt to be an imperfect mirror both of disciplinary concerns and of the social climate of the discipline.

The idea that the list was the discipline, albeit a warped version of it, also informed decisions of list members about active participation. Advertisements were sent to the list in the clear expectation that this was an appropriate way to publicize initiatives and opportunities to a broad systematics audience. Participation was seen as a service to the discipline, and in that sense continuous with other aspects of systematics as a vocation. In some cases contributors saw the list as a public record and felt compelled to offer information and corrections in order to correct misunderstandings or provide a more complete picture. A few members spoke of their list participation as fun, enjoying the intellectual stimulation of engaging in debate. Other individuals described inhibitions about contributing because of not wanting to make fools of themselves in front of peers, or spoke of correcting mistakes in private emails because they felt errors should not be shown up in public. Throughout the responses there was a sense of participation in the list as consequential for the discipline and for the reputation of members within the discipline, which was shared amongst many lurkers and active contributors.

My approach to the ethics of quoting material from the list throughout my research was informed by this understanding of what the list meant. Becoming aware that the list was 
viewed as a public forum, and that postings were viewed as consequential for reputation, I asked permission to use materials that I wished to quote, particularly where this involved archival materials and I might be inappropriately resurrecting pasts that those involved would prefer to forget. In practice I found that permission was always granted, and the approach often led to additional insights and discussions. The ethical commitment thus began as a duty and turned into an interesting and useful engagement.

The responses to my questions about list participation provided a rich set of perspectives to allow me to situate my observations. In numerical terms, however, they provided a very partial picture. Whilst the list membership at the time was around 1,400 , only 25 members responded to my appeal and engaged in dialogue about their participation. I therefore supplemented qualitative analysis of insights from participants with a simple quantitative assessment of list demographics compared with the demographics of the disciplinary population as a whole. This investigation was oriented towards an issue with particular significance for the systematics community: geographic inequalities.

Geography is heavily politicized in systematics. Whilst the less developed countries have much of the biodiversity, resources for systematic work are concentrated in the developed countries which often also have the specimen collections which relate to biodiversity in their former colonies. The internet has been promoted in some prominent initiatives as a means to enable resource-sharing to overcome some, at least, of the geographic inequalities. It therefore proves of particular concern to see how far participation in the Taxacom list reproduces or transcends existing geographic inequalities. 
The list organizer supplied me with the membership list broken down by country of origin. Disciplinary membership by country was available from the World Taxonomist Database maintained by ETI Bioinformatics (http://www.eti.uva.nl/tools/wtd.php). There were considerable imperfections in both of these data sets, and the conclusions could only be very broad approximations. Nonetheless, it was possible to calculate that the perception that US concerns were over-represented on the list was justified, since US subscribers were both numerically the largest group and a relatively high proportion of taxonomists from the US were subscribers. On this analysis the list is not reflective of the discipline across its full geographic distribution, but reproduces the inequalities which are a matter of concern in the global politics of biodiversity conservation. This observation offers a substantive insight into the tensions of developing e-sciences which adds to cautions about extent to which electronic communications can overcome existing inequalities in the science system (Barjak, 2006; Palackal, Anderson, Miller, \& Shrum, 2006). It also gives a further methodological caution into the use of mailing lists as disciplinary mirrors.

On the basis of these analyses I continued to use postings to the Taxacom list as a source of data on the concerns of a wider discipline, but with caution that every insight had to be tested for the extent to which it made sense according to other forms of disciplinary enactment and with a renewed realization that online ethnography should not be construed as being independent of geography. Mailing list observation can be a useful part of an ethnography of e-science, but it is valuable also to move the observation beyond list boundaries qualitatively and quantitatively, in order to situate list 
observations in other contexts relevant to the specific case. Sometimes this requires moving from online observations to offline, but sometimes an analytic mobility is required instead, shifting the frame in pursuit of the other meaning-making practices that a setting embeds and is embedded within. In the situation described here shifting the analytic frame back and forth between mailing list and discipline proved an effective and methodologically important strategy. Shifting the frame can help to bring into focus new connections to explore, and highlight issues that might otherwise be taken for granted. The next section illustrates this point by looking at a different kind of online/offline connection and highlighting again the ethnographic purchase offered by moving between the two.

\section{Web geographies and institutions}

Whilst some aspects of contemporary systematics were quite visible on the Taxacom list, other features of disciplinary life received far less prominence. In particular, the offline component of the ethnography was turning out to be centered around major institutions which featured very little, beyond occasional announcements, on the list. These institutions could however be observed quite readily on the web as individual sites which tended to figure prominently in search engine results. All of the major museums and herbaria had web sites, which often included a public-facing component with information for a general public and a research-oriented site with resources for other systematists. In this section I will describe the role that institutional web sites played in my ethnography, 
and in particular focus on the purchase offered by exploring the web landscape and connecting that with insights from institutional visits and literature analysis.

Institutional web sites were a valuable resource for tracking down potential interviewees, since I was able to find out who was involved in interesting initiatives and also to pinpoint key individuals to contact at the major institutions that I was going to be able to visit in person. In this sense, then, the ethnography habitually moved between online and offline. Whilst in other parts of the research I found email interviews an invaluable tool for opportunistic contacts and geographically dispersed informants, a face-to-face visit gave a sense of material culture and institutional location which enriched my understanding of the issues that shaped what was possible and desirable online. The routine movement between online and offline that my practice of recruiting interviewees entailed also gave me an experiential understanding of online communication as an embedded part of everyday life within systematics.

I also developed an experiential sense of the web landscape through my ongoing efforts to find relevant initiatives and work out they were related to one another. Some connections that I followed were literally hyperlinks, where one web site enabled me to move straight to another in a similar strategy to the snowballing that happens when one interviewee suggests another. In either case one applies a certain scepticism about whether the new link will turn out to be relevant for the project at hand. Again, moving from online to offline and back was a habitual, routine practice rather than a consciously adopted ethnographic strategy. It simply makes sense, in the contemporary media 
environment, to use the web as a tool for finding out about institutions, and in this sense there is no exoticized virtual sphere separate from the real (Miller \& Slater, 2000).

Development of appropriate institutional structures is acknowledged as a key challenge for e-science (David \& Spence, 2003). Everyday practices need to be understood as made possible by and also appropriated and made meaningful by an institutional environment. In systematics much of the work is organized around museums and herbaria which hold massive collections of preserved specimens. Systematists use the specimens within these collections as a resource for undertaking work on classification, and virtual resources are increasingly being developed in conjunction with specimen collections in order to give wider access to information about specimens. Exploring the connections which made sense of this activity became a major ethnographic puzzle, as it became clear that diverse strands of institutional funding structures, individual careers, political pressures, communication regimes and working practices with material artefacts helped to shape the development of virtual resources.

In addition to site visits and interviews an important source of insight into initiatives and their connections to institutions came from the web itself. By looking around at what is visible on the web and at the various ways in which different resources are linked it is possible to get a sense of it as a landscape, differentiated by prominent features and well trodden routes. Landscapes are heterogeneous, and populated by diverse features linked to one another in various ways. Thinking of the web as landscape in this way proved to be a useful ethnographic strategy. It prompted exploration of that landscape, its structures 
and its connections as a form of observation in its own right, in turn raising questions for interviews and observations to investigate how that landscape is constructed and made meaningful by participants in their ongoing work.

Exploring the landscape of the web can be organized in many ways. There is now a repertoire of specialist techniques using analysis of hyperlinks to expose features of the web landscape (Rogers, 2002; Rogers \& Marres, 2000; Thelwall, 2004). But without turning to such specialist approaches there are ways of using ready to hand tools like search engines as ethnographic devices to prompt questions to pursue. A search engine such as Google, does not of course give an objective portrayal of a territory. The Google algorithm favours particularly well-connected sites which many other sites link to (Brin \& Page, 1998). This is a problem if one seeks an objective statement of "what is out there". If looking for the major landmarks in a landscape, according to the people who live there, it becomes an invaluable asset. The search engine becomes a way of seeing the landscape as the web site developers inserting links see it. Formal link analysis tends to use its own web crawlers to collect links, since it is then possible to control for timing, depth and reach rather than relying on the vagaries of search engines with proprietary algorithms. In the case described here none of these issues seemed to significantly outweigh the requirement for a low-commitment exploratory approach which would facilitate development of questions about the web landscape.

Building on this ethnographic understanding of Google, a particular visualization tool became a useful component of the strategy for exploring the web landscape in this study. 
The tool that I used was the TouchGraph Google browser

(http://www.touchgraph.com/TGGoogleBrowser.html). This web-based service is a Java applet which piggybacks on the "related" facility in Google. The precise algorithm is not public, but it is generally understood that "related" sites in Google are those which not only share keywords, but are also co-linked by third party sites. This means, from an ethnographer's point of view, that we can think of them as being grouped together, for whatever reason, by people enough involved in a field to be producing web sites commenting on it.

The TouchGraph Google browser takes a seed URL and finds the top 30 related sites, then finds the top 30 related sites for each of those, and presents a visual representation of the resulting network of related sites. Any of the resulting nodes can be clicked on again to retrieve the site itself, or to reveal another network of related sites based on that new starting point. This became a way to explore the web landscape in terms of the kinds of initiative and institution which "went together" for whatever reason, prompting new questions to be explored in interviews and through further web explorations. In particular, it prompted questions about the response of institutions to the web, and the varying visibility of institutions and the initiatives which populated them. For example, a TouchGraph Google browser representation of the Natural History Museum in London (Figure 1) shows a peer network of museums, including other natural history museums (including the Museum Nationale d'Histoire Naturelle in Paris and the Smithsonian Institution in Washington) and other national museums (including the Science Museum and the National Portrait Gallery in London). Clearly, for some observers the Natural 
History Museum is a tourist site or a piece of national heritage, whilst for others it is a key location for study of natural history. The Natural History Museum's web presence orients to this diverse set of audiences: in interviews it was possible to discuss the conflicting pressures and complex responses which the different public personae of institutions impose for those implementing their web strategies.

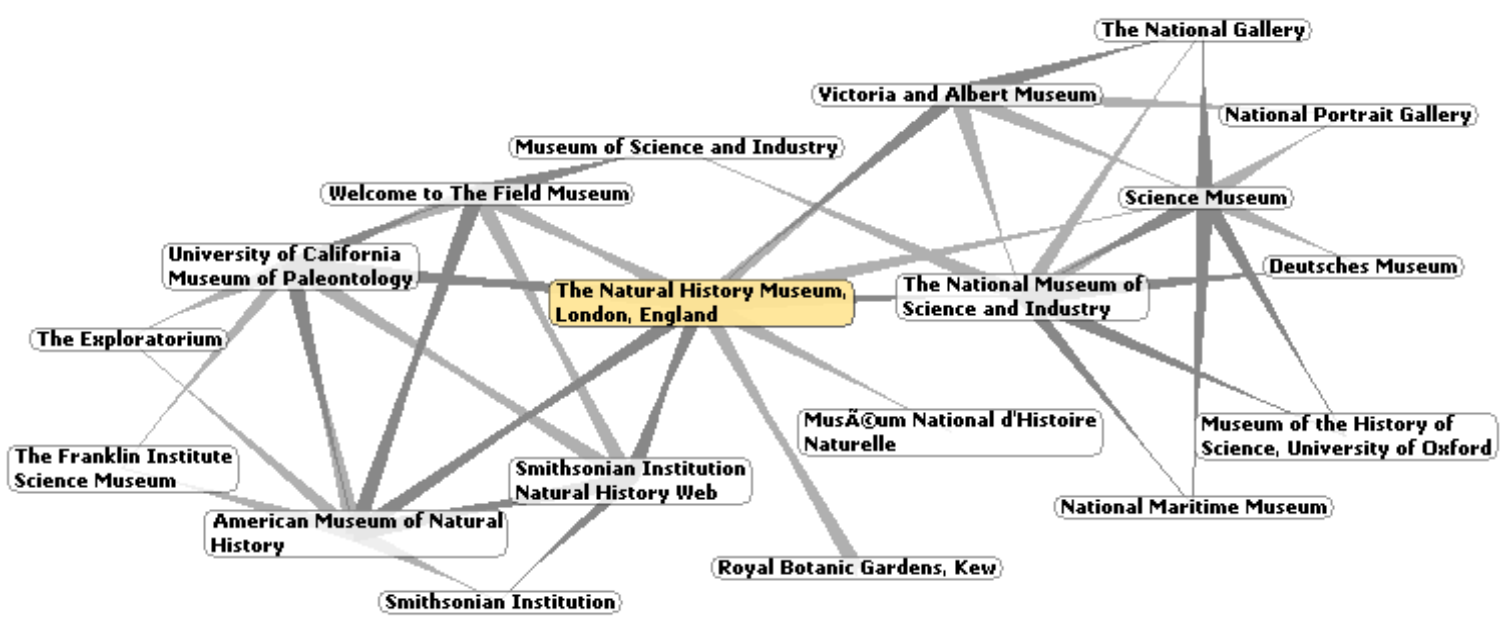

Figure 1. TouchGraph Google browser representation of a related sites network centered on the Natural History Museum, London

This picture constrasted dramatically with the related sites depicted by searching not for the institution itself, but for an initiative which is housed within that institution. Figure 2 shows the TouchGraph Google browser representation of the LepIndex project. LepIndex is a nomenclatural resource for Lepidoptera, created by digitizing a card index file held at the Natural History Museum in London (Beccaloni, Scoble, Robinson, Downton, \& Lucas, 2003). Despite being hosted on the museum's web site, as a flagship project for the museum, the LepIndex project appeared in a quite different web landscape. Its peer 
network, according to the TouchGraph Google browser, consists of other resources relating to Lepidoptera. This suggests that individual initiatives may have a visibility quite different to that of their host institutions, and that outsiders may orient to the initiative rather than the institution that houses it. This, in turn, raised a new set of questions about the way that institutions relate to initiatives, which provided questions to explore in interviews, and a new lens through which to read publicity and policy documents.

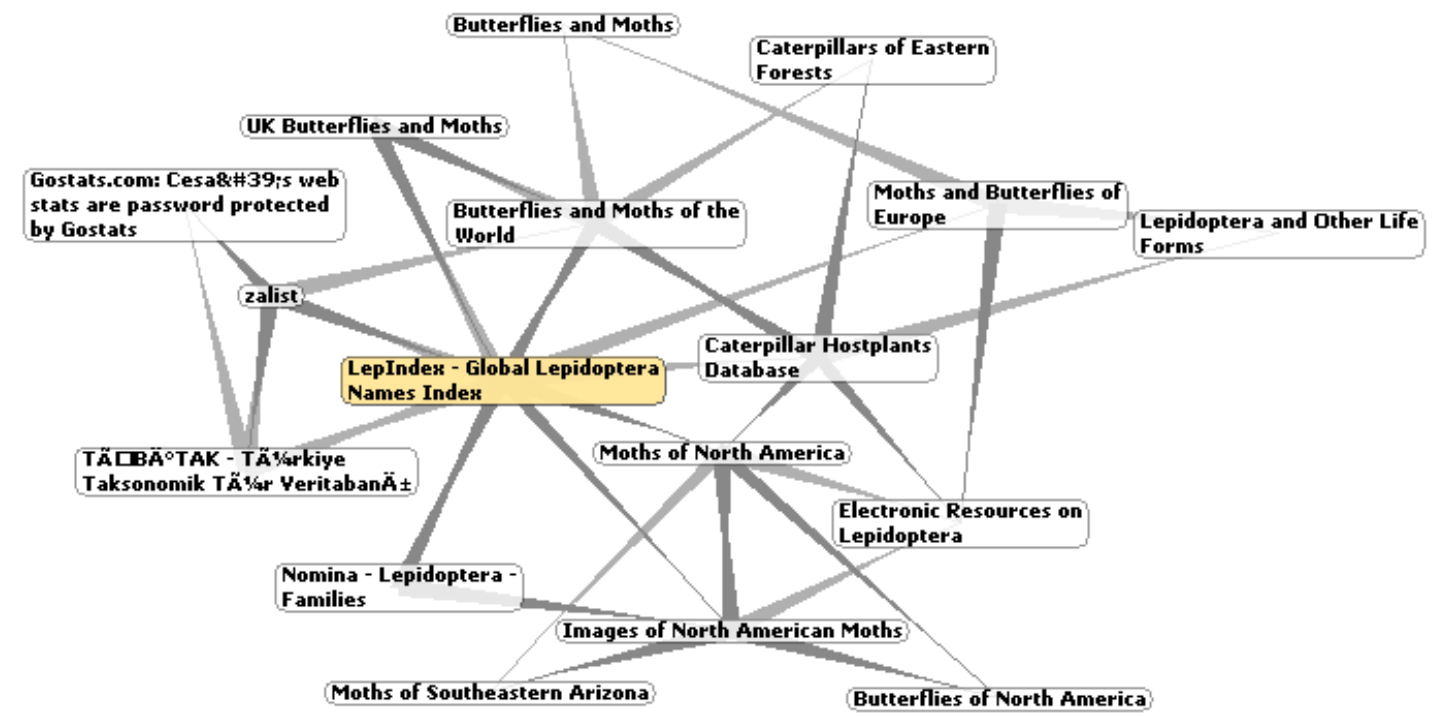

Figure 2. TouchGraph Google browser representation of a related sites network centered on the LepIndex initiative at the Natural History Museum, London

The ethnographer can, then, usefully see the web as a landscape to be explored, and aim to become sensitive to variations in the terrain. Just as there are maps to physical terrain, and just as those maps are not objective portrayals of territory but are instead selective in 
particular ways, so too do tools like Google, and the TouchGraph Google browser, give the ethnographer ways to navigate. Each of these ready to hand tools offers not a final answer, but a stimulus to further questions, offering ways to understand the landscape to be checked out from other viewpoints.

Ironically, the methodological recommendation to use ready to hand tools to explore the web landscape may apply more to areas outside of science than to other fields of escience. Systematics has been distinctive in its development of distributed databases which are openly accessible on the Internet. Most fields of e-science are much more protective of their data, and have tended to develop resources for use by closed communities. These fields of e-science may not therefore be as readily explored through the use of search engines and visualization tools, or indeed more formal link analysis. Ethnographic explorations of web landscapes need to be informed by an awareness of different linking practices and their meanings for authors (Beaulieu, 2005). The broader recommendation to explore online landscapes and consider the ways in which they reflect or reconstruct offline structures is, however, applicable across e-science.

\section{From policy to practice (and back)}

In exploring the institutional landscape of contemporary systematics it became clear that there was an over-riding commitment to digitization as something the community had to engage in. Many of those involved felt reservations about details of the implementation, or about the diversion of resources from other activities, but none were in any doubt that 
there was a prevailing climate in which they had to be seen to be engaged in digital initiatives. It therefore proved to be important within the overall ethnographic approach to explore where this perception arose, and how it informed practice. The ethnography thus also traveled from practices within systematics institutions and in online forums into the policy domain and back again, exploring the features of that domain to which systematists oriented themselves and the ways in which systematists both represented and were represented by policy.

One key aspect of the policy domain to which interviewees oriented themselves was the Convention on Biological Diversity. This convention, signed at the Rio Earth Summit in 1992, recognized that a lack of taxonomic information was an obstacle to efforts to conserve biological diversity. Countries who signed up to the convention were required to share with others their expertise and resources in systematics, thus addressing the geographical inequalities which left some countries rich in biodiversity poor in the resources to understand and conserve it. As signatories to the convention nation states are the site of formal reporting and assessment under the convention. However, the systematics institutions play a key role in informing their government's responses to the convention and accepting responsibility for meeting its requirements.

Within the UK, the capacity to meet requirements under the Convention on Biological Diversity was examined by a House of Lords Select Committee on Science and Technology report published in 2002. This report provided a significant resource for the ethnography, offering as it did a set of explicit recommendations for systematics and 
involving along the way considerable comment on the role of information and communication technology in the future of the discipline. Such reports are, of course, not transparent reflections of the policy process, nor are they taken by readers as directives for future action. The complex status of the report was an issue for discussion in interviews with some of the key players who informed the report and institutional responses to it. The report is no more straightforwardly representative of policy than the Taxacom list is representative of the discipline. Nonetheless, a report of this kind does again provide some kind of map of the territory and the concerns which populate it. The published report preserves the spoken and written evidence submitted to the committee as well as the recommendations, and as such provides a rich resource for exploring how those concerned identified themselves and portrayed their concerns in the public arena.

For the purposes of the methodological discussion in this paper the main point to make about the report is its reliance on information and communication technologies, which were proposed as a solution to the problems which it identified for systematics in terms of efficiency and reputation. The point is made in the executive summary of the report:

We highlight the importance of digitising the systematic biology collections, which will both increase accessibility of these data and help to update the archaic image of systematic biology. (Select Committee on Science and Technology, 2002a: 5). 
Throughout both the main body of the report and the various pieces of evidence considered, it becomes clear that the current climate is suffused with the expectation that information and communication technologies will be used with transformative effect. Interviewees confirmed the report as an expression of a policy climate of which they were aware and which they had to some extent helped to shape, and which in turn informed their assessment of desirable actions to take. Their assessment of appropriate courses of action was suffused by disquiet about the reputation of the discipline and concerns to sustain its fundability. Interviewees, particularly those responsible for shaping institutional policies, recognized the climate as favouring the adoption of new technologies. Nonetheless, this did not mean wholesale adoption of every suggestion that the report made. The House of Lords report made particular mention of a radical vision for the transformation of systematics, based around adoption of the web for publication of consensus taxonomies (C. Godfray, 2002; H. C. J. Godfray, 2002). Whilst systematics institutions publicly aligned themselves with the move towards digital solutions they used the platform of the report to reject this radical solution and promote existing initiatives. The report provided an opportunity to stress progress in the favoured direction, portraying existing initiatives as appropriate and carefully designed, rather than over-cautious or lacking in vision. When challenged to introduce radical transformation systematists have been able to argue that they have transformed, but in appropriate forms that express considerable continuity with disciplinary tradition.

The report therefore portrays a climate which favours certain kinds of initiative according to the technologies which they deploy. Analysis of the evidence informing the report and 
interviews with systematists about their response to the issues demonstrated that this climate was experienced both as a pressure and as a strategic opportunity. Whilst systematics institutions may have felt that they had no choice but to develop digital resources, they also found their prominent political position an opportunity to portray existing activities in a favourable light and to pursue possibilities for new funding. This situation is highly specific to systematics, but has a broader methodological significance. Within an ethnographic strategy for understanding e-science it is important to consider how expectations about online activities are being shaped, and also to pursue the way that online activities are presented for other audiences. Just because an activity happens online does not mean that it is oriented to, or wholly consumed by, an immediate online audience. The potential transformation of science through the introduction of e-science has been a prominent policy concern and a widely discussed expectation in recent years. It is particularly important, then, to make connections between expectations and practices in e-science, to explore the way that expectations shape what it is possible and desirable for scientists to do online, and to consider the ways in which online activities are repackaged as they travel into other domains.

\section{Conclusion: e-science and ethnographic evolution}

The ethnographic approach which I describe above was highly specific to the cultural context in question. Systematics is an unusual scientific discipline in a number of regards, including its international political profile, its distinctive institutional arrangements, its material culture and its orientation towards legacy literature. These distinctive qualities 
have shaped an approach to information and communications technologies which is in dialogue with e-science, but diverges from the stricter definitions of that phenomenon. The outcomes of this ethnography in terms of the particular response of systematics to information and communication technologies, and the form of the ethnography in terms of the sites visited and the connections between them are highly specific. Nonetheless, the overall approach of exploring the ways in which diverse forms of activity are connected to and mutually inform one another, could be a fruitful one to generalize to other situations.

The approach that I describe had key components which could inform a provisional ethnographic strategy for e-science. An ethnographer could usefully explore: online forums and the extent to which they represent and transform disciplinary identity; institutional structures and their representation in web landscapes; policy directions and their consumption and reproduction in and of on-the-ground activities. Navigating a way through these various connections offers the potential to develop a rich and rounded approach to understanding the experience of e-science. Indeed, many domains of contemporary life would be amenable to such an approach, appropriately adapted to explore the various strands of interpretation and expectation that make sense of particular technological choices. In the approach proposed here different sites emerge as important and inform one another in various ways, and whilst sometimes the online/offline distinction will be apparent, in other cases it will seamlessly be crossed. Taking this approach offers a way of remaining sceptical about the possibility of spatial transformation (Green, Harvey, \& Knox, 2005), and in particular about the status of the 
offline in relation to the online. In this regard it is in sympathy with Orgad's (2005) approach to the combination of online and offline observations as mutually informative with neither consistently contextualizing the other, and Constable's (2003) integration of online observations into a more widely constituted ethnographic inquiry.

It is debatable whether the methodological approaches described here are particularly novel in themselves, beyond a willingness to be agile in the face of the challenges of new phenomena. As Jankowski and van Selm suggest, many methodological responses to new information and communication technologies have been of the order of minor adjustments rather than radical rethinking (Jankowski \& van Selm, 2005). Beaulieu too identifies the transfer of ethnography to the internet as a matter of, in the main, "gentle worries, milder claims and subtler adjustments" (Beaulieu, 2004), rather than radical shifts in practice or in epistemological substance. It is quite plausible to anchor current adaptations of ethnography in disciplinary legacies even whilst highlighting innovation, and there is a methodological rationale for consciously adopting an ambiguous stance. Remaining agnostic about the transformative capacities of new technologies suggests caution about any prospect of designing appropriate ethnographies in advance, and instead increases focus on adaptive ethnography which explores connections and boundaries experientially. This form of ethnography is simultaneously old and new, being grounded in a tradition of emergence and adaptation.

\section{Acknowledgments}


This research was funded by the UK Economic and Social Research Council under grant number R000271262. I am grateful to Nicholas Jankowski and two anonymous referees for comments which helped in revising the paper. In particular I am grateful for the recommendation which drew Leander and McKim (2003) to my attention.

\section{References}

Amit, V. (Ed.). (2000). Constructing the Field: Ethnographic Fieldwork in the Contemporary World. London: Routledge.

Barjak, F. (2006). From the "analogue divide" to the "hybrid divide": the internet does not ensure equality of access to information in science. In C. Hine (Ed.), New Infrastructures for Knowledge Production: Understanding E-Science (pp. 233 245). Hershey, PA: Idea Group.

Beaulieu, A. (2004). Mediating ethnography: objectivity and the making of ethnographies of the Internet. Social Epistemology, 18(2-3), 139-163.

Beaulieu, A. (2005). Sociable hyperlinks: an ethnographic approach to connectivity. In C. Hine (Ed.), Virtual Methods: Issues in Social Research on the Internet (pp. 183 198). Oxford: Berg.

Beaulieu, A., \& Simakova, E. (2006). Textured connectivity: an ethnographic approach to understanding the timescape of hyperlinks. Cybermetrics, 10(1). Retrieved 8 June 2006 from http://www.cindoc.csic.es/cybermetrics/articles/v10i1p6.html

Beccaloni, G. W., Scoble, M. J., Robinson, G. S., Downton, A. C., \& Lucas, S. M. (2003). Computerising unit-level data in natural history card archives. In M. J. 
Scoble (Ed.), ENHSIN: The European Natural History Specimen Information Network (pp. 165 - 176). London: Natural History Museum.

Bowker, G. (2000). Biodiversity datadiversity. Social Studies of Science, 30(5), 643-684.

Brin, S., \& Page, L. (1998). The Anatomy of a Large-Scale Hypertextual Web Search

Engine. Retrieved 8 June 2006, from http://www-

db.stanford.edu/ backrub/google.html

Burawoy, M. (Ed.). (2000). Global Ethnography: Forces, Connections and Imaginations in a Postmodern World. Berkeley: University of California Press.

Collins, H. (2004). Gravity's Shadow: the Search for Gravitational Waves. Chicago: University of Chicago Press.

Collins, H. M. (1975). The seven sexes: a study in the sociology of a phenomenon, or the replication of experiments in physics. Sociology, 9(2), 205-224.

Collins, H. M. (1985). Changing Order: Replication and Induction in Scientific Practice. London: Sage.

Constable, N. (2003). Romance on a Global Stage: Pen Pals, Virtual Ethnography and "Mail Order" Marriages. Berkeley: University of California Press.

David, P. A., \& Spence, M. (2003). Towards Institutional Infrastructures for E-Science: the Scope of the Challenge. Retrieved 8 June 2006, from http://www.oii.ox.ac.uk/resources/publications/OIIRR_E-Science_0903.pdf

Epstein, S. (1996). Impure Science: AIDS, Activism and the Politics of Science. Berkeley, CA: University of California Press.

Galison, P. (1997). Image and Logic: material culture of microphysics. Chicago: University of Chicago Press. 
Godfray, C. (2002). How might more systematics be funded? Antenna, 26(1), 11-17.

Godfray, H. C. J. (2002). Towards taxonomy's 'glorious revolution'. Nature, 420(6915), 461-461.

Green, S., Harvey, P., \& Knox, H. (2005). Scales of place and networks - An ethnography of the imperative to connect through information and communications technologies. Current Anthropology, 46(5), 805-826.

Gupta, A., \& Ferguson, J. (Eds.). (1997). Anthropological Locations: Boundaries and Grounds of a Field Science. Berkeley, CA: University of California Press.

Heath, D. (1998). Locating genetic knowledge: Picturing Marfan syndrome and its traveling constituencies. Science Technology \& Human Values, 23(1), 71-97.

Heath, D., Koch, E., Ley, B., \& Montoya, M. (1999). Nodes and queries - Linking locations in networked fields of inquiry. American Behavioral Scientist, 43(3), 450-463.

Henke, C. R. (2000). Making a place for science: The field trial. Social Studies of Science, 30(4), 483-511.

Hess, D. J. (2001). Ethnography and the development of science and technology studies. In P. Atkinson, A. Coffey, S. Delamont, J. Lofland \& L. Lofland (Eds.), Sage Handbook of Ethnography (pp. 234 - 245). Thousand Oaks, CA: Sage.

Hey, T., \& Trefethen, A. E. (2002). The UK e-Science core programme and the Grid. Future Generation Computing Systems, 18(8), 1017 - 1031.

Hilgartner, S. (1995). Biomolecular databases: new communication regimes for biology. Science Communication, 17(2), 240-263. 
Hilgartner, S., \& Brandt Rauf, S. I. (1994). Data access, ownership, and control - toward empirical studies of access practices. Knowledge-Creation Diffusion Utilization, 15(4), 355-372.

Hine, C. (2000). Virtual Ethnography. London: Sage.

Hine, C. (Ed.). (2006). New Infrastructures for Knowledge Production: Understanding EScience. Hershey, PA: Information Science Publishing.

Howard, P. N. (2004). Embedded media: who we know, what we know, and society online. In P. N. Howard \& S. Jones (Eds.), Society Online: the Internet in Context (pp. 1 - 27). Thousand Oaks, CA: Sage.

Jankowski, N. W., \& van Selm, M. (2005). Epilogue: methodological concerns and innovations in Internet research. In C. Hine (Ed.), Virtual Methods: issues in social research on the Internet (pp. 199 - 207). Oxford: Berg.

Knorr-Cetina, K. (1981). The Manufacture of Knowledge: an Essay on the Constructivist and Contextual Nature of Science. Oxford: Pergamon Press.

Knorr-Cetina, K. (1999). Epistemic Cultures: how the Sciences make Knowledge. Cambridge, MA: Harvard University Press.

Latour, B., \& Woolgar, S. (1986). Laboratory Life: the Construction of Scientific Facts (2 ed.). Princeton: Princeton University Press.

Leander, K. M., \& McKim, K. K. (2003). Tracing the everyday "sitings" of adolescents on the Internet: a strategic adaptation of ethnography across online and offline spaces. Education, Communication and Information, 3(2), 211 - 240.

Lynch, M. (1985). Art and Artifact in Laboratory Science: a Study of Shop Work and Shop Talk in a Research Laboratory. London: Routledge and Kegan Paul. 
Marcus, G. (1995). Ethnography in/of the world system: the emergence of multi-sited ethnography. Annual Review of Anthropology, 24, 95-117.

Marcus, G. (1998). Ethnography Through Thick and Thin. Princeton: Princeton University Press.

Martin, E. (1994). Flexible Bodies: Tracking Immunity in American Culture from the Days of Polio to the Age of AIDS. Boston: Beacon Press.

Martin, E. (1998). Anthropology and the cultural study of science. Science Technology \& Human Values, 23(1), 24-44.

Miller, D., \& Slater, D. (2000). The Internet: an Ethnographic Approach. Oxford: Berg. Mody, C. C. M. (2005). The sounds of science: listening to laboratory practice. Science Technology \& Human Values, 30(2), 175 - 198.

Orgad, S. S. (2005). From online to offline and back: moving from online to offline research relationships with informants. In C. Hine (Ed.), Virtual Methods: Issues in Social Research on the Internet (pp. 51 - 66). Oxford: Berg.

Palackal, A., Anderson, M., Miller, B. P., \& Shrum, W. (2006). Gender stratification and e-science: can the internet circumvent patrifocality? In C. Hine (Ed.), New Infrastructures for Knowledge Production: Understanding E-Science (pp. 246 271). Hershey, PA: Idea Group.

Rogers, R. (2002). Operating issue networks on the web. Science as Culture, 11(2), 191 214.

Rogers, R., \& Marres, N. (2000). Landscaping climate change: a mapping technique for understanding science and technology debates on the World Wide Web. Public Understanding of Science, 9, 1 - 23. 
Thelwall, M. (2004). Link Analysis: an Information Science Approach. Amsterdam: Elsevier Academic Press.

Zenzen, M., \& Restivo, S. (1982). The mysterious morphology of immiscible liquids: a study of scientific practice. Social Science Information, 21(3), 447-473. 